\title{
Outcome of Tuberculous Meningitis in Adults
}

\author{
Salma Haji
}

\begin{abstract}
OBJECTIVE: To determine the frequency of mortality and morbidity of TBM patients in our part of world and predictors of mortality.

METHODOLOGY: Prospective observational study. Study was conducted at neuro medicine ward at JPMC Karachi from March 2015 to March 2018. Total 110 patients were included. All diagnosed patients of TBM above 12 years of age were included. Mortality and Morbidity of the patients were recorded. The predictors of mortality were also assessed. Patient with other CNS disease were excluded from study.

RESULTS: Male patients were predominantly $(60 \%)$ involved. Mortality was $10 \%$. Complication in TBM in descending order were Hydrocephalus $60 \%$, Cranial nerve palsies $\mathbf{4 0} \%$, Hyponatremia $\mathbf{4 0} \%$, Stroke $30 \%$, Papilledema $20 \%$, Myelitis $10 \%$, Ataxia $10 \%$, Acute seizure $10 \%$ and Dementia $10 \%$. Hydrocephalus, use of mechanical ventilation, TLC more than 9000 were present $100 \%$ in all 11 dead patients. At the end of standard duration of treatment $70 \%$ of patients had complete recovery without residual deficit. $20 \%$ patient had residual deficit and $(10 \%)$ died.

CONCLUSION: Mortality of TBM patient is very high in stage III presentation, Hydrocephalus, Hyponatremic and use of ventilation are predictor of mortality. Hydrocephalus, Cranial nerve palsies, Hyponatremia and Stroke are common complication and less common complications are Myelitis, Papilledema, Acute seizure and Dementia.
\end{abstract}

KEYWORDS: Tuberculous Meningitis, Adults, Outcome.

This article may be cited as: Haji S. Outcome of Tuberculous Meningitis in Adults. J Liaquat Uni Med Health Sci. 2019;18(01):28-33. doi: 10.22442/jlumhs.191810597

\section{INTRODUCTION}

Tuberculous meningitis (TBM) is the most common form of central nervous system disease which has very high morbidity and mortality. Various case series showed a mortality rate of $7 \%-100 \%$ in developed countries and $69 \%$ in underdeveloped countries ${ }^{1}$. TBM is common in female and children. TBM was sub -acute disease, symptoms were present for ten days to nine months. Low grade fever, malaise, headache, dizziness and vomiting may persist for few days then patient can develop altered mental status, stroke, hydrocephalus and cranial nerves palsies. Seizures are uncommon presentation of TBM in adult ${ }^{2}$. Neck stiffness and fever maybe absent. Neurological complications may occur like mental retardation, hydrocephalus, cranial nerves palsies, stroke, seizures and coma ${ }^{3,4}$. Diagnosis of TBM can be difficult. Moderate CSF leucocytosis and presence of local deficit increase the possibility of TBM.CSF PCR has shown sensitivity of $87 \%-95 \%{ }^{5}$. MRI can detect hypodensities due to cerebral infarct, cerebral edema and it is superior to computed tomography (CT) for evaluating the brain stem. It is good for detection of infarct due to $\mathrm{TBM}^{6}$. Mycobacterium tuberculosis bacilli enter the host by inhalation then escalates lungs and can spread in other organs and brain parenchyma resulting in formation of small subpialfoci of caseous lesions. These are called rich foci. Rich focus rupture into subarachinoid space cause meningitis, deeper in brain parenchyma cause abscess. Abscess can rupture into ventricle. Exudate infiltrate the cortical or meningeal blood vessel producing obstruction. Meningitis accounts for abnormality of cranial nerve 3,6 and 7 and hydrocephalus due to obstruction of basilar cisterns, neurological pathology is produced by adhesion formation, and encephalitis. Papilloedema may cause visual loss. Cranial nerve six is affected mostly by TBM followed by cranial nerve 3, 4, 7 and then commonly 2, 8, 10, 11 and 12 nerves. Monoplegia, hemiplegia and tetraplegia is due to vasculitis. Lastly fibroid degeneration of adventitia within small arteries and veins produces aneurysm and focal hemorrhages in Pakistan hydrocephalis is most common complication $(20 \%)$ and mortality is $6 \%{ }^{7}$. Prognosis of TBM depends on clinical stage of disease, variation of host immunity and virulence of mycobacterium. Predictors of prognosis of TBM are hydrocephalus, infarction, and tuberculoma. Study shows significant variables were age, stage of disease, focal weakness, cranial nerve palsy and hydrocephalus. ${ }^{8}$ Early treatment improve outcome of TBM. Thus treatment is 
needed when clinical features and CSF findings are suggestive of TBM. Treatment regimen of TBM consist of $\mathrm{INH}$, rifampicin, pyrazinamide, ethambutol for two months and ten months of INH and rifampicin. Adjunctive corticosteroid therapy reduced the severe complications ${ }^{9}$.

Morbidity and mortality is very high (69\%) in TBM ${ }^{1}$. The rational of this study is to find out the factors which are responsible for high mortality and morbidity so that strategies can be made to prevent or decrease the complication of TBM and to know the exact frequency of complication of TBM in our setup. The objective of study is to find out the frequency of mortality and morbidity of TBM in our setup.

\section{METHODOLOGY}

It was prospective observational study conducted at Neuro medicine department ward 28 JPMC Karachi from March 2015 to March 2018 for three years. Total 110 patient of TBM were included in study, 66 patients were male and 44 patients were female. All diagnosed cases of TBM above 12 years of age of both males and females were included. Patients with other brain diseases like viral encephalitis, intracranial bleed, cerebral malaria and stroke were excluded.

All patients of TBM above 12 years of age were admitted through OPD or emergency department of JPMC was enrolled in study. The detail history, examination, lab investigation, findings were recorded in performa.

Investigations like complete blood count, liver function test, random blood sugar, electrolytes, mantoux test, chest X-ray and fundoscopy were carried out. Computed tomography (CT) brain scan with contrast and CSF analysis with AFB straining and culture were carried out for diagnosis. The necessary investigation like lymph node biopsy in an accessible part and MRI were carried out for diagnosis of TBM.

On the basis of clinical presentation patients of TBM were classified in three stages. Stage I included nonspecific symptoms and signs such as headache, vomiting, fever and anorexia without impaired level of consciousness. Stage II was altered level of consciousness or focal neurological deficit without coma. Stage III included unconsciousness with severe neurological deficit and seizures. All patients during stay were assessed for complications and managed accordingly.

Patients were followed in wards and in OPD to note the results of treatment. Morbidity and mortality were recorded in performa. Other variables like age, sex, residence etc. were recorded. At the cessation of follow up, the outcome was determined in term of recovery, recovery with residual disability and mortality.

Data was analyzed by SPSS version-24. Continuous variables like age, sex, duration of symptoms were analyzed by standard deviation. Categorical variable like frequency of morbidity and mortality was expressed in frequency and proportion.

Ethical consideration- Confidentiality of the data of patient was maintained strictly. An informed consent was taken from all patients for lumber puncture.

\section{RESULTS}

About $60 \%$ patients were $20-40$ years of age, $15 \%$ of patients were more than 40 years of age and $25 \%$ were above 12 year and below 20 year of age. Out of 110 patients, 77 patients developed complication or death. Mortality rate was $10 \%$ and $70 \%$ patient completely cured with ATT and $20 \%$ end up with residual deficit. Leading complication of TBM was hydrocephalus $(60 \%)$ than second most common complication was cranial nerve palsies (40\%). Abducent nerve and optic nerve were involved dominantly. $6^{\text {th }}$ cranial nerve was usually recovered with treatment but the prognosis of $2^{\text {nd }}$ was poor. Hyponatremia was in $40 \%$ patients of TBM and treated successfully in most of the patients stroke occurred in $30 \%$ patients of TBM and was also recovered in 22 patients recovered with treatment. Papilledema was in $20 \%$ patient. Acute seizure and myelitis were also noted in TBM patients. Dementia was rare.

Hydrocephalus was mostly associated with cranial nerve palsies, hyponatremia, stroke and other complication of TBM. Myoclonic jerk, papilledema and choroid tubercle was less common presentation.

Mortality predictors were extreme of age more than 60 years. In 11 patients mechanical ventilator was used and all expired. All 11 patient who expired were presented in stage III, septicemia (TLC more than 9000), hydrocephalic and hyponatremia.

$30 \%$ patients have a history of contact with tuberculosis patients and $40 \%$ patients were found to have tuberculosis infection affecting other organs at presentation. Associated pulmonary TB was most common (30\%). Other site extra meningeal TB was abdominal $3 \%$ lymphnoned $4 \%$ and spinal $2 \%$.

ATT with adjunctive steroid were started in all patients. (Table I and II). 
TABLE I:

MORBIDITY AND MORTALITY OF TBM PATIENTS

\begin{tabular}{|l|c|c|}
\hline Morbidity And Mortality & $\begin{array}{c}\text { No. of } \\
\text { patients }\end{array}$ & Percentage \\
\hline Death & 11 & $10 \%$ \\
\hline Hydrocephalus & 66 & $60 \%$ \\
\hline Cranial nerve palsies & 44 & $40 \%$ \\
\hline Hyponatremia & 44 & $44 \%$ \\
\hline Stroke & 33 & $30 \%$ \\
\hline Papilledema & 22 & $20 \%$ \\
\hline Myelitis & 11 & $10 \%$ \\
\hline Ataxia & 11 & $10 \%$ \\
\hline Dementia & 11 & $10 \%$ \\
\hline Acute seizure & 11 & $10 \%$ \\
\hline \multicolumn{2}{|l|}{ Total no. of patients of TBM were 110} \\
\hline
\end{tabular}

TABLE II: CRANIAL NERVE PALSIES IN TBM

\begin{tabular}{|l|c|c|}
\hline \multicolumn{1}{|c|}{ Cranial nerve palsies } & $\begin{array}{c}\text { No. of } \\
\text { patients }\end{array}$ & $\begin{array}{c}\text { Relative } \\
\text { percentage }\end{array}$ \\
\hline Left IV N palsy & 2 & $4.54 \%$ \\
\hline Left VI N palsy & 2 & $4.54 \%$ \\
\hline Bilateral II N palsy & 7 & $15.90 \%$ \\
\hline Bilateral II and III N palsies & 2 & $4.54 \%$ \\
\hline II and VII N palsies & 2 & $4.54 \%$ \\
\hline IV,VII,IX,X N palsies & 4 & $9.10 \%$ \\
\hline II Nerve palsy & 2 & $4.54 \%$ \\
\hline III nerve palsy & 2 & $4.54 \%$ \\
\hline Left III N palsy & 4 & $9.10 \%$ \\
\hline Bilateral VI N palsy & 8 & $18.18 \%$ \\
\hline III,IV and VI N palsy & 3 & $6.81 \%$ \\
\hline VI and VII N palsy & 2 & $4.54 \%$ \\
\hline VII N palsy & 4 & $9.10 \%$ \\
\hline Total Number of cranial nerve palsies patients were 44 \\
\hline
\end{tabular}

\section{DISSCUSION}

The objective of study was to find out the incidence of mortality and morbidity in our setup and to know the predictors of mortality. The mortality in this study was $10 \%$. Mortality rate was comparatively low than other local studies. Mortality rate range in local study was $22-25 \%{ }^{10}$. While study abroad showed mortality range was $6.9 \%-7.7 \%{ }^{11}$. This low mortality rate as compared to local study as in our study may be due to the reason of early presentation of TBM. 90\% of TBM patient presented in stage I \& II and only $10 \%$ of patients presented in stage III. Predominantly patients presented in stage II are in accordance with most of the other studies ${ }^{12}$. Another study ${ }^{18}$ showed the predictor of mortality were age more than 60 years, use of mechanical ventilator, presentation of patient in stage III. In this study the relative low mortality of TBM patient may be due to the early presentation of patient (stage I\&II) and young age of patient. Mortality can be reduced further if high index of suspicion among our physician is made in our setup to early diagnose the TBM.

Hydrocephalus was common complication in TBM. It was usually communicative $(73.01 \%)$ and noncommunicative $(26.9 \%)^{3,4}$. In other international study with TBM patient's hydrocephalus was found in $62 \%$ adult and it was $87 \%$ in children ${ }^{14}$. In this study hydrocephalus was $60 \%$. It is almost same as in national and international studies. All the 11 patients who died had hydrocephalus so it was proved to be a strong predictor of mortality, $16.66 \%$ (11/66) of hydrocephalic patient died and other survived with treatment, 40 patients managed conservatively and cured. Prognosis of hydrocephalus was good because they presented in stage I and II. In international study patients of hydrocephalus who required surgery mortality was $45.1 \%$ and disability was up to $55 \%{ }^{15}$. In this study in twelve patient of hydrocephalus shunt surgery was done. In most of the patients grade of hydrocephalic patient is best. It has been found that patient in severe hydrocephalus presentation developed severe disability ${ }^{4}$. In this study 10/11 expired with hydrocephalus were presented in stage III. In stage I presentation hydrocephalus is less common for early diagnosis of TBM patient can prevent hydrocephalus leading to mortality and disability. Hydrocephalic patient in grade I and II should be managed medically and should be monitored to detect any improvement. Shunt operation should be offered to the patient in whom medical treatment has failed. Shunt has usually additional mortality in TBM patient. Hydrocephalus leads to mortality and disability in TBM patient. In grade I and II of hydrocephalus when edema and exudate is present in the ventricle and choroid plexuses is inflamed than prognosis of medical treatment was good because brain is not much damaged. Hydrocephalus in advanced grade had a lot of scarring of subarachnoid space and bad prognosis.

In this study cranial nerve palsies was $40 \%$. Optic and abducent nerve were predominantly involved, $60 \%$ of cranial nerve palsies were associated with 
hydrocephalus. There was $32 \%$ cranial nerve palsy in study conducted in South Africa ${ }^{16}$. Most of the cranial nerve palsies in this study recovered with treatment but second nerve palsy left permanent blindness. Presentation with Cranial nerve palsies differentiates TBM from acute bacterial meningitis. Cranial nerve palsies were usually due to hydrocephalus, ischemic infarction and tuberculoma. Mortality was high if cause is ischemic infarction ${ }^{17}$. In this study cranial nerve palsy was slightly higher than other studies.

Hyponatremia in this study was $40 \%$. Hyponatremia in international study is $40-50 \%$ in $\mathrm{TBM}^{15}$, so it was same as in international study. Hyponatremia was independent predictor of death. Diagnosis mimic with TBM hyponatremia includes central salt wasting syndrome (CSWS), syndrome of inappropriate secretion (SIADH) and adrenal insufficiency. The available evidence suggests that cause of hyponatremia in TBM is CSWS. $34 \%$ patient of hydrocephalus in this study developed hyponatremia and 7 patients died. We treated the hyponatremia patient with $3 \%$ saline. Mostly patient recovered, 7 hyponatremia patients died which showed hyponatremia is a predictor of mortality in TBM.

Stroke in this study was $30 \%$. Stroke in TBM in international study was $15-57 \%^{18}$, so stroke in our study is higher than international study. Majority of the stroke may be asymptomatic. MRI is most sensitive in detecting acute and chronic stroke. Stroke in TBM are multiple, bilateral and located in the basal ganglia. Cortical stroke can also occur due to involvement of proximal portion of middle, interior and posterior cerebral arteries as well as in supra clinoid portion of internal carotid and basilar arteries which are documented in MRI. Arteritis was common than infarction in $\mathrm{TBM}^{18}$. Corticosteroid and aspirin reduced the mortality and the incidence of stroke ${ }^{18}$. We used steroid and aspirin in stroke patient. Most of our patients recovered.

Bilateral papilledema and loss of vision was common presentation in children in TBM. Our 23\% patients have papilledema and blindness most of the patient recovered with ATT and steroid so it is proved papilledema is also common in adult.

Myelitis in TBM is a rare complication. Our 11 patients had myelitis and recovered with ATT. Ataxia and dementia were also found in our study it was less common as compared to other studies. Acute seizure occurs in about $5 \%$ of adult ${ }^{2}$. Status epilepticus and seizure may complicate TBM. Recurrent seizures are common. In this study $10 \%$ patients developed who were successfully treated with benzodiazepine and with phenytoin.

In this study $90 \%$ patients presented in stage I and II and mean age was 31 year. Male was slightly predominant. Same result was found in other study ${ }^{18}$. TBM was associated with $40 \%$ in extra CNS tuberculosis and was associated $30 \%$ with pulmonary Koch. In study conducted in civil hospital Karachi. TBM had $30 \%$ association with extra CNS TB. International study shows the same result ${ }^{19,20}$.

Predictors of mortality in this study were mechanical ventilation $(100 \%)$, hydrocephalus $(100 \%)$, hyponatremia $(87.5 \%)$, leucyositis (100\%), presentation in stage III $(87.5 \%)$ and age more than 60 years $25 \%$, another study reported that age more than 60 and mechanical ventilation are predictors of mortality ${ }^{19}$. Our study showed stage III presentation, use of mechanical ventilation, hyponatremia; hydrocephalus and septicemia are strong predictors of mortality.

It is recommended that high mortality in TBM can be prevented only by early diagnosis of TBM patient in stage I disease. So always make high index of suspicion in nonspecific presentation in TBM patients. Early lumber puncture for CSF analysis and early MRI brain can be done for early diagnosis of TBM to prevent the TBM patient to enter in to stage II and III. In this way we can prevent hyponatremia, hydrocephalus and use of mechanical ventilator which can lead to mortality and morbidity. If this complication already developed then aggressive management of this complication should be done to reduce the mortality. BCG vaccination in children should be enforced in Pakistan. Public awareness program to reduce the contact with known patients of TB Should be addressed. Reduction of TBM related deaths can be made with the initiation of anti TBM regimen before the onset of coma ${ }^{21-23}$ So diagnosis and treatment of TBM in stage I can reduce the mortality and morbidity. Limitation In this study was exclusion of children. Mortality may be more if we include children. Some patients came in coma and expired undiagnosed may be case of TBM.

\section{CONCLUSION}

Tuberculosis meningitis is a frequently reported problem in our part of world. Hydrocephalus along with other sequel like cranial nerve palsies, hyponatremia, stroke, dementia, myelitis, acute seizure is common complications. Early lumbar puncture for CSF analysis and MRI brain can diagnose the TBM in stage $1^{\text {st }}$ and sequel of TBM can be prevented if early diagnosis is made. Predictors of mortality are hydrocephalus, use of mechanical ventilation, TLC more than 9 thousand, hyponatremia, presentation of TBM in stage three in this study. Early diagnosis with intensive investigation on the basis of suspicion of TBM can prevent mortality and disability. 


\section{ACKNOWLEDGMENT}

I would like to thank Dr. Shaukat Ali for his guidance in scientific advice.

Conflict of Interest: No conflict of interest.

\section{REFERENCES}

1. Chiang SS, Khan FA, Milstein MB, Tolman AW, Benedetti A, Starke JR, et al. Treatment outcomes of childhood tuberculous meningitis: a systematic review and meta-analysis. Lancet Infect Dis. 2014; 14(10): 947-57. doi:10.1016/ S1473-3099(14)70852-7.

2. Schoeman JF, Janse van Rensburg $A$, Laubscher $J A$, Springer $P$. The role of aspirin in childhood tuberculous meningitis. J Child Neurol.2011; 26 (8):956-62. doi: 10.1177/0883073811398132.

3. Rizvi I, Garg RK, Malhotra HS, Kumar N, Sharma E, Srivastava C, et al. Ventriculo-peritoneal shunt surgery for tuberculous meningitis: a systematic review. J Neurol Sci. 2017;375:255-63. doi:10.1016/j.jns.2017.02.008.

4. Rajshekhar V. Surgery for brain tuberculosis: a review. Acta Neurochir (Wien). 2015; 157 (10):1665-78. doi: 10.1007/s00701-015-2501-x.

5. Sharma K, Sharma A, Pallab R, Kumar SS. Multiplex PCR for rapid diagnosis of tuberculous meningitis. J Neuro. 2011; 258(10): 1781-87.

6. Sharma RM, Pruthi N, Arimappamagan A, Somanna S, Devi BI, Pandey P. Tubercular meningitis with hydrocephalus with HIV co-infection: role of cerebrospinal fluid diversion procedures. J Neurosurg. 2015; 122(5):1087-95. doi:10.3171/2014.12.JNS14257.

7. Shaikh MA, Shah M, Channa F. Criteria indicating morbidity in tuberclous meningitis. JPMA. 2012; 62(11):1137-9.

8. Moghtaderi A, Alavi-Naini R, Rashki S. Cranial nerve palsy as a factor to differentiate tuberculous meningitis from acute bacterial meningitis. Acta Med Iran. 2013; 51(2):113-8.

9. Thuong N, Heemskerk D, Tram T, Thao LTP, Ramakhrishnan L, Ha VTN, et al. Leukotriene A4 hydrolase genotype and HIV infection influence intracerebral inflammation and survival from tuberculous meningitis. J Infect Dis. 2017; 215 (7):1020-8. doi:10.1093/infdis/jix050.

10. Fazel PA, Makki KU, Haroon H, Soomro IB, Afzal $U$. Clinical spectrum and outcome of patients with tuberculous meningitis. Med Channel. 2006; 12: 21-23.

11. Akkerman OW, Odish OF, Bolhuis MS, de Lange
WC, Kremer HP, Luijckx GJ, et al. Pharmacokinetics of bedaquiline in cerebrospinal fluid and serum in multidrug-resistant tuberculous meningitis. Clin Infect Dis. 2016; 62(4):523-4. doi:10.1093/cid/civ921.

12. Zhang X, Falagas M, Vardakas K, Wang R, Qin R, Wang $J$, et al. Systematic review and meta-analysis of the efficacy and safety of therapy with linezolid containing regimens in the treatment of multidrug-resistant and extensively drug-resistant tuberculosis. J Thorac Dis. 2015; 7 (4):603-15.

13. Sobri M, Merican JS, Nordiyana M, Valarmathi S, Al-Edrus SA. Neuroimaging features of tuberculous meningitis. Med J Malaysia. 2006; 61 (1): 36-40.

14. Marais S, Meintjes G, Pepper DJ, Dodd LE, Schutz C, Ismail Z, et al. Frequency, severity, and prediction of tuberculous meningitis immune reconstitution inflammatory syndrome. Clin Infect Dis. 2013; 56(3):450-60. doi:10.1093/cid/cis899.

15. Misra UK, Kalita J, Bhoi SK, singh RK. A study of hyponatremia in tuberculous meningitis. $J$ Neurol Sci. 2016; 367:152-7. doi:10.1016/ j.jns.2016.06.004.

16. van Well GT, Paes BF, Springer $P$, Roord JJ, Donald PR, van Furth AM, et al. Twenty years of pediatric tuberculous meningitis: a retrospective cohort study in the western cape of South Africa. Pediatrics. 2009; 123(1):e1-8.

17. Garg RK, Malhotra HS, Kumar N, Uniyal R. Vision loss in tuberculous meningitis. J Neurol Sci. 375:27-34. doi: 10.1016/j.jns.2017.01.031

18. Misra UK, Kalita J, Maurya PK. Stroke in TBM. J Neurol Sci. 2011; 303(1-2): 22-30. doi: 10.1016/ j.jns.2010.12.015.

19. WHO. World Health Organization Global Health Observatory Database. Tuberculosis Cases Mortality and prevalence. (Online) 2011. (Cited 2011 April 10). Available from https://www.who. int/gho/en/

20. Qureshi HU, Merwat SN, Nawaz SA, Rana AA, Malik A, Mahmud MK, et al. Predictors of inpatient mortality in 190 adult patients with tuberculous meningitis. J Pak Med Assoc. 2002; 52(4): 15963.

21. Heemskerk AD, Bang ND, Mai N, Chau T, Phu N, Loc $P$, et al. Intensified Antituberculosis Therapy in adults with Tuberculous Meningitis. $\mathrm{N}$ Engl $\mathrm{J}$ Med. 2016; 374:124-134. doi: 10.1056/ nejmoa1507 062 . 
22. Van Toorn R, Schaaf HF, Laubscher JA, van Elsland SL, Donald PR, Schoeman JF. Short intensified treatment in children with drug-susceptible tuberculous meningitis. Pediatr Infect Dis J. 2014; 33(3):248-52. doi:10.1097/ INF.0000000000000065.

23. Ruslami R, Ganiem AR, Dian S, Apriani L,
Achmad $\mathrm{TH}$, van der Van AJ, et al. Intensified regimen containing rifampicin and moxifloxacin for tuberculous meningitis: an open-label, randomised controlled phase 2 trial. Lancet Infect Dis. 2013; 13(1):27-35. doi:10.10 16/ S1473-3099 (12)70264-5.

\section{橉}

AUTHOR AFFILIATION:

Dr. Salma Haji

Neurologist

University of Karachi, Sindh-Pakistan.

Email: salmahajineuro@gmail.com 